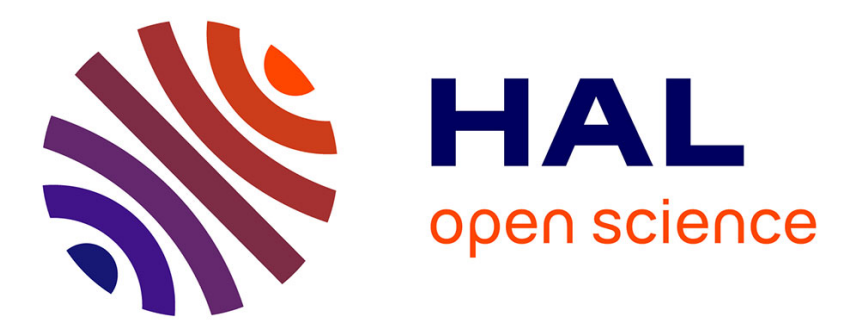

\title{
Les travailleurs immigrés du bâtiment entre discrimination et précarité. L'exemple d'une activité externalisée: le ferraillage
}

Nicolas Jounin

\section{- To cite this version:}

Nicolas Jounin. Les travailleurs immigrés du bâtiment entre discrimination et précarité. L'exemple d'une activité externalisée: le ferraillage. La Revue de l'IRES, 2006, 10.3917/rdli.050.0003 . hal01822292

\section{HAL Id: hal-01822292 \\ https://hal.science/hal-01822292}

Submitted on 16 Aug 2018

HAL is a multi-disciplinary open access archive for the deposit and dissemination of scientific research documents, whether they are published or not. The documents may come from teaching and research institutions in France or abroad, or from public or private research centers.
L'archive ouverte pluridisciplinaire $\mathbf{H A L}$, est destinée au dépôt et à la diffusion de documents scientifiques de niveau recherche, publiés ou non, émanant des établissements d'enseignement et de recherche français ou étrangers, des laboratoires publics ou privés. 


\title{
Les travailleurs immigrés du bâtiment entre discrimination et précarité. L'exemple d'une activité externalisée : le ferraillage
}

\author{
Nicolas JOUNIN *
}

\section{Introduction}

En mai 2004, le terminal 2E de l'aéroport de Roissy s'effondre, tuant six personnes. Le secteur du bâtiment retrouve la une des médias, et le mot « ferraillage » est popularisé car l'activité qu'il désigne est mise en cause dans la catastrophe ${ }^{1}$. Autre mise en cause : celle de la sous-traitance. Quelques semaines avant l'accident, l'architecte du terminal déclarait : «En général, je constate que la qualité de mise en œuvre risque à tout moment de se dissoudre dans la mauvaise qualité des méthodes et le renouvellement permanent des équipes $»^{2}$. Mais un reportage du journal télévisé de France 2 entend rassurer le public en présentant l'un des chantiers modèles de l'heure, le viaduc de Millau. «En cas d'accident, c'est le constructeur qui sera responsable. Aussi a-t-il choisi de diminuer au maximum le nombre de sous-traitants », commente le journaliste, avant de laisser la parole au « directeur compagnie Eiffage », qui explique : «La multiplicité des sous-traitants donne évidemment des exigences supplémentaires en termes de coordination, de surveillance. Là, ce sont au maximum les entreprises d'Eiffage qui réalisent le chantier et par conséquent nous avons une bien meilleure maîtrise de la

* Docteur en sociologie (Paris VII - Unité de recherche Migrations et société).

1. Sur le chantier, le ferraillage est l'activité qui, au minimum, consiste à poser les armatures qui soutiendront la structure en béton, et peut aller jusqu'à leur façonnage et leur assemblage. Mais ces deux dernières tâches sont de moins en moins exécutées sur place. C'est pourquoi, pour décrire le ferraillage dans son ensemble, il faudrait parler des usines de préfabrication, auxquelles les entreprises de pose sont adossées : techniquement, commercialement (l'offre de l'une dépend de l'autre, et il peut y avoir des alliances), et même parfois financièrement (par des participations ou des concentrations). Cependant, je n'évoquerai ici que l'activité de chantier.

2. Cybertbtp, $26 / 05 / 2004$. 
qualité et de la sécurité » ${ }^{1}$. On en déduit, a contrario, que la tendance générale à l'externalisation, qui semble n'épargner que les ouvrages d'art, ne répond pas à une recherche de qualité et de sécurité, voire se satisfait de moindres performances à ce niveau. Ce qui laisse supposer l'existence d'avantages compensant ces carences - peut-être en matière de coût du travail et de discipline des travailleurs.

Autre événement - plus discret - touchant à la sous-traitance, en juillet 2004, à Roissy toujours : l'un des nombreux chantiers près de l'aéroport est bouclé par une centaine de policiers. Sur le chantier, une PME spécialisée dans la pose d'armatures (le ferraillage) utilise une quinzaine d'ouvriers. Dix d'entre eux disparaissent du chantier à la suite du contrôle de police, soit enfuis, soit arrêtés. Ils sont sans papiers. Le patron de la PME (appelons-la FF) est entendu par la police mais pas inquiété. L'entreprise générale qui a sous-traité la pose d'armatures à FF l'est encore moins. L'agence d'intérim qui employait les ferrailleurs pourrait être davantage menacée, mais depuis lors elle a toujours pignon sur rue. Pour parer à cette défection soudaine, FF appelle d'autres agences d'intérim pour obtenir de nouveaux ferrailleurs. De nouveaux ouvriers arrivent en nombre chaque matin, et ils sont presque aussi nombreux à être renvoyés chaque soir, car les intérimaires détachés ne font pas l'affaire. Paradoxe apparent : les ferrailleurs disparus avaient beau être intérimaires et sans papiers, précaires parmi les précaires, ils travaillaient avec FF pour certains depuis des années. Avec eux, le chef de chantier avait pu relativement stabiliser un savoir-faire et une routine de travail, qui étaient désormais perdus. Les «mauvaises méthodes » et le « renouvellement permanent des équipes » (selon les mots de l'architecte de Roissy) guettaient à nouveau : dangers toujours recréés par le système d'emploi précaire en place dans le gros œuvre, et toujours compensés par des aménagements informels de ce système.

Les discours sur le secteur du bâtiment empruntent en général à des imageries contrastées. D'un côté, on y voit le règne de la précarité, submergé par les liens froids et impersonnels du marché, et par une ferme discipline de chantier. De l'autre, ce sera plutôt le paradis des liens chauds, de la camaraderie aux enclaves ethniques, des filières de recrutement aux tribus. On trouve assurément de la précarité dans le ferraillage : il s'agit d'un métier sous-traité depuis une vingtaine d'années, un métier de PME qui s'affrontent sur un marché très concurrentiel, et où le taux de recours à l'intérim oscille entre 60 et $75 \%$... Et pourtant, plusieurs phénomènes étonnent : comment expliquer notamment qu'au milieu de cet univers de concurrence universelle, les ferrailleurs soient de manière si écrasante des immigrés maghrébins ? Pourquoi ne sont-ils pas également portugais ou afri-

1. JT de 20h, France 2, 24/05/2004. 
cains ? Pourquoi «si tu es coffreur, il faut parler portugais, si tu es ferrailleur, il faut parler arabe », ainsi que le souligne un ferrailleur en riant? Qu'est-ce que ce particularisme recréé au cœur des mécanismes de marché a priori universalistes?

Ce sont les questions qui ont guidé ma recherche, et les réponses sont résumées ici à travers le cas du ferraillage. Il s'agit de montrer les balancements permanents de la gestion de la main-d'œuvre, entre précarité pour tous et protections sélectives. L'entrée par le drame de Roissy - qui demeure exceptionnel - indique le risque qui surplombe cette gestion, celui d'une exécution de l'ouvrage (trop) défectueuse. Selon son conducteur de travaux, le patron de FF « se lève tous les matins en se demandant comment ça se fait qu'il n'y ait pas plus de bâtiments qui se cassent la figure en France ». Comment peut-on en arriver à se poser ce genre de question ? Et pourquoi la prédiction qu'elle contient, malgré tout, ne se réalise-t-elle pas? Je ne prétends pas répondre ici à ces interrogations, mais il faut les garder en tête car elles constituent la trame cachée de la gestion de la main-d'œuvre qui va être décrite.

Il s'agira, dans un premier temps, de retracer l'histoire de l'externalisation du ferraillage; dans un deuxième temps, d'établir le profil actuel des ferrailleurs, intérimaires et étrangers, c'est-à-dire doublement vulnérables, en le resituant dans les modalités historiques d'utilisation de main-d'œuvre immigrée dans le bâtiment. Certes les employeurs utilisent des ouvriers précaires, mais pas n'importe lesquels : dans un troisième temps, il faudra décrire les mécanismes qui fabriquent une homogénéité ethnique des ferrailleurs. On verra dans un dernier temps que ces mécanismes s'inscrivent dans une nécessité (du point de vue de la production) de réinjecter des loyautés pour pallier les insuffisances de la discipline par la précarité.

La méthodologie choisie n'est pas favorable à la profondeur historique : privilégiant une analyse synchronique des chantiers ici et maintenant, j’ai principalement usé de l'observation participante (occupant des postes d'ouvriers durant neuf mois sur des chantiers franciliens du gros œuvre). Mais l'observation a été prolongée par une cinquantaine d'entretiens, notamment avec des ouvriers et des responsables d'entreprises du ferraillage. Une autre difficulté surgit alors : l'histoire récente du ferraillage est dure à écrire. Pour des raisons qui cependant constituent déjà une information : d'abord, les recherches sur le bâtiment se sont taries à l'aube de sa mise en sous-traitance ${ }^{1}$;

\footnotetext{
1. Lorsque la sociologie du travail se développe durant les «Trente glorieuses », elle laisse de côté le bâtiment, trop archaïque à son goût. II faut attendre la crise du fordisme et le début des années 1980 pour que le bâtiment soit étudié de manière approfondie. Autour du Plan construction architecture du ministère de l'Equipement, plusieurs chercheuses lui consacrent des recherches qui restent des références (par exemple Campinos-Dubernet, 1984 ; Campagnac, 1992). Mais à partir de la seconde moitié des années 1990, le passage de ces chercheuses à d'autres objets d'étude ne s'est pas accompagné d'une relève.
} 
ensuite, la spécialisation d'entreprises dans ce métier n'a pas encore appelé une autonomisation dans les institutions qui l'encadrent (codage professionnel et statistique, syndicats, etc.) ; enfin, les vieux ferrailleurs susceptibles de témoigner du passé sont rares. C'est pourquoi ce texte, s'il s'appuie pour décrire le présent sur mon expérience de quatre mois dans deux entreprises de ferraillage (qu'on appellera FE et FF) et sur les discussions et entretiens réalisés avec des ouvriers, est tributaire pour le passé des témoignages des cadres et chefs d'entreprise (une administrative, des chefs de chantier, et le patron de FE, des chefs de chantier et un conducteur de travaux de FF). Sources similaires donc, mais variées malgré tout : certains interviewés se trouvent dans une trajectoire sociale ascendante, d'autres régressent ou ont l'impression de régresser au sein de leur métier ; FE est florissante, tandis que FF risque de fermer bientôt.

\section{Encadré 1 .}

\section{Méthodologie de l'enquête}

La recherche de doctorat (Jounin, 2006), dont est issu cet article, est le résultat d'une enquête ethnographique, basée essentiellement sur la méthode de l'observation participante. Pendant neuf mois, j'ai travaillé comme intérimaire ou stagiaire ouvrier sur divers chantiers, dans diverses entreprises (entreprises générales et sous-traitantes en ferraillage) et divers métiers (manœuvre, coffrage, ferraillage) ${ }^{1}$. Dès le départ, afin d'intégrer la sous-traitance et l'intérim dans une logique d'ensemble de gestion de la main-d'œuvre, il a été décidé de considérer que le chantier, et non l'entreprise, devait être l'unité pertinente de l'enquête. Si l'on élimine quelques courtes missions d'intérim, j'ai observé six chantiers, pendant trois à huit semaines chacun. Sur deux d'entre eux, introduit comme stagiaire, j'étais connu comme observateur ; sur les autres, seuls quelques proches collègues ont su mes objectifs.

J'ai rédigé quotidiennement un journal de terrain. Peu de notes sont retranscrites ici, mais la plupart des constats (proportion d'intérim, absentéisme par exemple) en sont issus. Les entretiens concernant le ferraillage ont été conduits avec des ouvriers ou des responsables des entreprises où j'ai moi-même travaillé. Non seulement l'observation préalable facilitait la prise de contacts, mais elle déterminait également la trame des entretiens, car il était alors possible de remplacer des questions générales («comment se déroule le travail ? », " employez-vous des sans-papiers ? »...), qui risquaient de susciter des réponses creuses ou évasives, par des questions précises fondées sur des événements particuliers (" que s'est-il passé avec tel chef ? ", " que s'est-il passé après le contrôle de police sur le chantier ? »...). Dès lors, comme dans le travail journalistique, des recoupements sont possibles, et l'on peut considérer les entretiens autrement que comme la simple expression d'expériences et de sentiments personnels : ils 


\section{- -}

sont aussi des témoignages permettant d'établir des faits. Dans la mesure où les personnes interviewées ont des liens entre elles, les entretiens ont donc des fonctions comparables à l'observation : dessiner une configuration d'acteurs et de relations (Burawoy, 2003 [1998]).

Et surtout, si peu de notes de terrain sont reproduites dans cet article, en revanche la problématique est directement issue des découvertes faites pendant l'observation participante. Ainsi, avant cette étape, j'envisageais d'étudier la précarisation du travail et le racisme frappant les travailleurs (ou une partie d'entre eux), sans forcément trouver une articulation logique entre les deux. Les ouvriers étaient vus principalement comme des victimes, dans un rôle passif. Au cours de l'observation cependant, j'ai consigné les détournements, les pauses cachées, les désertions, les petites révoltes des ouvriers que je côtoyais, voulant rendre une sorte d'hommage à ces résistances. II ne s'agissait alors que d'hommage, et je ne leur donnais aucune portée analytique. Or, si les résistances de ce type ne semblent en l'état actuel porteuses d'aucune transformation d'envergure, il est apparu au fur et à mesure qu'elles constituent un problème quotidien pour les hiérarchies, contraintes de mettre en place des stratégies correspondantes afin de « limiter les dégâts ». C'est ainsi que s'est développée la problématique des loyautés dans le travail, qui sera plus précisément examinée au terme de cet article.

1. S'y ajoutent trois mois de formation en coffrage et ferraillage en lycée professionnel.

\section{Le ferraillage, histoire d'une externalisation}

La prise en charge du ferraillage se trouvait, au début des années 1980, partagée entre deux types d'entreprises : sidérurgiques et de construction. Les grandes entreprises sidérurgiques assuraient la pose des armatures sur les gros chantiers, tandis que les entreprises générales de bâtiment s'en chargeaient sur les plus petits. D'après tous les témoignages, l'apparition de petites entreprises de ferraillage résulte des projets d'externalisation des grands groupes (et non l'inverse : les projets qui découleraient de l'opportunité soudaine de confier le travail à de nouveaux opérateurs). Les petits patrons du ferraillage sont d'anciens cadres du métier ; ils obtiennent leurs premiers marchés par ces mêmes groupes qu'ils quittent, ou grâce aux relations qu'ils y ont tissées. Ainsi, FE s'est montée dans la deuxième moitié des années 1980, avec deux associés qui se sont connus sur des gros chantiers en Arabie saoudite. C'est le plus jeune des deux, aujourd'hui seul patron de FE, et que l'on appellera M. Dauby, qui raconte : 
«- Moi, j’ai fait que deux boîtes. J'ai fait dix ans chez Usinor-Sacilor, dans leurs différentes filiales. Et puis après j'ai fait 17, 18 ans dans ma société. (...) C'est le patron que j'avais en Arabie, qui m'a proposé de m'associer à lui.

- Et comment ça s'est passé?

- (...) Il est venu me voir, il m'a proposé de m'associer avec lui, et de monter une société en Ile-de-France. (...) Lui, il apportait son expérience, son relationnel. Et puis moi j'apportais ma jeunesse, mon dynamisme... (...)

- Vous avez, eu des clients tout de suite, grâce à son relationnel?

- Oui, on a travaillé... On n'est pas partis, vraiment, de notre société, mal. On est partis en travaillant, un peu, en partenariat avec eux. Donc en définitive, on est partis, et on était en même temps sous-traitants de notre ancienne société.

- Parce que eux-mêmes, c'était une période où ils commençaient à sous-traiter le ferraillage?

- (...) Ils étaient quand même intéressés de travailler avec des sociétés qui avaient des charges réduites, qu'ils vendent leurs aciers, et que nous on exécute la pose en sous-traitance. (...) On a été quand même beaucoup appuyés par les commerciaux d'une des sociétés de notre ancien groupe, où on avait trois commerciaux. (...) Je veux dire, on n'aurait jamais pu se payer ça.».

Tandis que pour M. Dauby, monter cette entreprise représente un envol, la création de FF est plus tortueuse. Son fondateur, M. Harmann, est l'ancien dirigeant d'une grande entreprise de ferraillage, Unifer, filiale d'Usinor, qui a dû fermer. Mais, dans le récit de Patrick Renoir, conducteur de travaux de FF depuis les débuts de l'entreprise (et gendre de M. Harmann), on voit qu'un certain nombre d'événements et de relations sont similaires dans les histoires de FE et FF :

«- [M. Harmann] était patron d'une grosse entreprise de pose en France qui s'appelait Unifer, et il était aussi patron d'une usine en France qui s'appelait Unime. Et... pardon : il était gérant de la pose et patron de l'usine. Tout ça sous le couvert d'Usinor-Sacilor, grosse filiale de l'Etat français. Et ce qui s'est passé, c'est qu'il y a eu un patron d'Usinor-Sacilor qui a été mis en place. Un mec qui connaissait absolument rien au métier, et qui parlait que de profit, que de rentabilité. Donc qui dit rentabilité dit "trop de dépenses", trop de dépenses dit "trop de salaires, donc trop de bonhommes". (...) Et il y a eu des grèves monstrueuses en 84, 85. Des grosses grèves qui ont mis un genou à terre à Unime. Là-dessus, le père Harmann, avec ses collaborateurs, a réussi à remonter un petit peu tout ça. Et l'autre derrière, a fini de casser le truc, en arrêtant les chantiers en 
Arabie saoudite qui ramenaient beaucoup d'argent. Et donc, comme là, M. Harmann avait donné sa parole aux délégués, et que l'autre, à chaque fois, allait contre sa parole, du jour au lendemain il a donné sa démission. (...) Et il y a un monsieur qui s'appelle M. Ledragon, de chez Bâtiment SA, qui a eu un gros chantier, le chantier LeCentre à la Défense. Et donc M. Ledragon avait travaillé très très longtemps avec M. Harmann. L'ancienne époque de la parole donnée, de "on se tape dans la main et tu me donnes le chantier". Et donc il est allé voir plusieurs personnes, notamment M. Harmann, pour lui dire : "Tiens, tu me fais mon chantier LeCentre", qui représentait 5000 tonnes d'armature en douze mois. C'est un record absolu, c'est... On l'a jamais refait en vingt ans. Donc le père Harmann à l'époque, il avait pas d'entreprise, il avait rien du tout. Donc il fait son entreprise pendant LeCentre. Les bureaux de chantier, c'était son entreprise. Et il a appelé ses anciens collaborateurs d'Unime qui ont tout de suite lâché et qui sont venus bosser avec lui. (...) Et puis là-dessus s'est greffée une autre entreprise, qui avait un chantier parallèle, qui s'appelait Construction Cie. Et à l'époque Construction Cie, était plus que grandissante. Et donc Construction Cie, l'autre entreprise, a dit à notre entreprise : "Tiens, on va te filer du boulot". Et FF, pendant dix ans, n'a pas fait de commercial. Jamais. ».

A l'origine, ni les patrons de FE ni ceux de FF n'ont eu à faire de « commercial », c'est-à-dire démarcher des entreprises. Les clients étaient déjà là : c'étaient les anciens patrons ou clients, ceux d'avant l'externalisation. Dans un premier temps, les hommes, les acteurs du ferraillage n'ont pas changé ; seules les structures évoluaient. Le métier éclata en petites entités : usines d'armatures d'un côté, entreprises de pose de l'autre. Le développement de la préfabrication en usine raréfia les emplois de ferrailleurs, tandis que le recours croissant à l'intérim ${ }^{1}$ accentuait la concurrence entre ceux qui restaient. Les ferrailleurs, qui avaient jusque-là un profil similaire aux autres ouvriers des entreprises du gros œuvre (majoritairement des immigrés arrivés pendant les «Trente glorieuses »), sont devenus de jeunes immigrés précai-

1. En région parisienne, les entreprises de travail temporaire (ETT) spécialisées dans le gros œuvre se concentrent autour des gares du Nord et de l'Est à Paris. Les majors (Manpower, Adecco...) y tiennent une part minoritaire. Ce sont les petites (une ou deux agences) et les moyennes (jusqu'à dix agences) structures qui fournissent le gros des intérimaires en gros oeuvre. Des unes aux autres, les différences de taille ne semblent pas déterminantes. Certes, des économies d'échelles sont réalisables dans le travail administratif (paies, déclarations, etc.). Mais les entretiens réalisés avec des commerciaux et les discussions avec des intérimaires laissent penser que l'échelon de gestion est celui du « commercial » plus que de l'agence. Le commercial (souvent la commerciale) a " ses " clients comme il a " ses » intérimaires : c'est en essayant d'emmener les uns et les autres qu'il quitte une ETT pour s'embaucher dans une nouvelle. 
res (voir plus bas). Qu'est-il advenu des anciens ferrailleurs qui ont perdu leur emploi ou fui les bas salaires qui s'imposaient ? C'est difficile à dire.

Sur la région parisienne, les entreprises moyennes de pose, du gabarit de FE ou FF, sont une dizaine. Leurs effectifs embauchés sont ceux de petites entreprises : FE avait 24 embauchés en 2004, FF en avait 40. Mais toutes deux faisaient travailler au moins cent personnes, ce qui veut dire 60 à $75 \%$ d'intérimaires. La mise en sous-traitance a fait éclater les acteurs du ferraillage en petites structures en concurrence les unes avec les autres. Malgré quelques alliances pour de gros chantiers, malgré les liens privilégiés que chacune de ces PME peut entretenir avec telle ou telle entreprise générale, il reste que les entreprises de ferraillage se sont livrées une concurrence féroce qui laisse penser que plusieurs d'entre elles disparaitront, plutôt à court qu'à long terme. M. Dauby, le patron de FE, analyse ainsi cette concurrence, dont son entreprise s'est plutôt bien sortie :

«- Il est certain que c'est un métier qui est moins porteur financièrement qu'il l'a été il y a dix ans. (...)

- Ça, ça s'explique comment?

- Ça s'explique, c'est parce qu'on a une concurrence... C'est devenu un métier de PME. Avant on était à l'ombre des grands groupes. Donc les grands groupes faisaient des marges plus importantes, nous on vivait très bien de marges un peu plus faibles à l'ombre de ces grosses sociétés-là. Aujourd'hui qu'on n'est que des PME, on s'est quand même beaucoup tapé dans la gueule. (...) Donc quelque part, pour essayer de s'en sortir, on a commencé à tirer un peu sur tout, et sur un petit peu la rémunération. Quand t'es en situation où tu commences à développer qu'un point ou un demi point de marge sur des chiffres d'affaires qui sont quand même pas négligeables, t'es regardant à tout. ».

A entendre M. Renoir, conducteur de travaux de FF, l'avenir de sa société est assez sombre. Il évoque un chantier, déficitaire comme beaucoup d'autres, en l'occurrence de 200000 francs :

«- T’en fais cinq comme ça dans l'année, t’as perdu un million de francs, boum. Un million de francs dans l'année, t'as trois millions de francs de réserve, dans trois ans t'es mort. C'est aussi simple que ça. C'est malheureux, mais c'est la vérité. (...) Sur dix entreprises de pose parisiennes... dignes de ce nom : qui sont capables de faire des chantiers avec deux trois grues. (...) Sur les dix, t'en as trois, allez quatre, qui sont en train de mourir. ».

Lorsqu'elles étaient dans le giron des groupes, les entreprises de ferraillage n'étaient pas soumises aussi brutalement à la loi du marché. Leurs commandes étaient garanties par les grandes sociétés dont elles dépendaient, ou 
bien leurs résultats entraient dans une stratégie d'ensemble, où les coûts immédiats n'étaient qu'une variable parmi d'autres. Une fois indépendantes, ces entreprises se voient contraintes de se plier au seul critère qui règle désormais l'attitude de leurs clients : le prix le plus bas possible. Elles répercutent sur leurs salariés la minimisation des tarifs et l'incertitude sur la pérennité des commandes, en cherchant à faire baisser les salaires et accroître la précarité.

Dans ces entreprises, on ne trouve pas de syndicats. De manière plus générale, sur les chantiers, les syndicalistes sont rares. Dans un secteur où le patronat a toujours mal vu leur implantation à l'intérieur de l'entreprise (les syndicats n'étant appréciés que pour la gestion d'organismes paritaires [Tallard, 1986 : 16-22]), les délégués ont du mal à s'imposer. L'aversion historique du patronat du bâtiment envers les syndicats s'associe aujourd'hui à la dilution de la notion d'employeur, à travers les réseaux de sous-traitance et d'intérim. Il faut ajouter que lorsque des syndicalistes sont présents sur un chantier, il s'agit de salariés de l'entreprise générale, qui ne sont pas toujours vus comme des alliés par les salariés des entreprises sous-traitantes, car ils sont susceptibles de porter des revendications, au niveau de l'organisation ou de la sécurité, qui amènent à un surcroît de travail pour les sous-traitants (dans la mesure où ces derniers doivent s'adapter aux changements avec des moyens inchangés).

\section{Utiliser des immigrés précaires pour des formes d'emploi précaires...}

Entre 60 et $75 \%$ des effectifs des entreprises sous-traitantes ferraillage sont intérimaires, et davantage encore pendant les périodes de pointe, tandis que, dans les effectifs de l'entreprise générale, la proportion va plutôt de 25 à $60 \%$. Non seulement la sous-traitance n'est pas une alternative à l'intérim, mais, dans le cas du ferraillage, la sous-traitance appelle l'intérim. Pour des raisons somme toute logiques :

1) En dépit des primes de congés payés et de précarité, en dépit également de la marge de l'agence d'intérim, les intérimaires coûteraient moins cher que les embauchés, d'après certains responsables. Il est malaisé d'attester cela, précisément parce que les ferrailleurs embauchés sont devenus l'exception. Historiquement, en tout cas, l'intérim a été l'agent de la baisse des rémunérations, ainsi que de leur écrasement, c'est-à-dire qu'il y a peu de différence entre les bons et les mauvais ferrailleurs - ce qui a tendance à dégoûter les premiers. Aujourd'hui néanmoins, maintenant que la baisse des tarifs est acquise, un ferrailleur embauché coûterait-il moins cher ? Ce n'est pas sûr, mais l'intérim a d'autres avantages, le premier d'entre eux étant le pou- 
voir de renvoyer les ouvriers du jour au lendemain (grâce à la pratique des agences d'intérim, illégale mais routinière, qui consiste à ne pas faire signer de contrat, ou seulement en fin de mission). Cela permet également à l'entreprise de ne pas dépasser le seuil fatidique des 50 salariés.

2) Les entreprises de ferraillage doivent faire face au problème de variabilité dont se sont débarrassés leurs commanditaires en sous-traitant. Avec de moindres outils : alors que les entreprises générales pouvaient favoriser la polyvalence de leurs ferrailleurs afin de les occuper à d'autres tâches (maçonnerie, coffrage) en cas de creux, les entreprises spécialisées dans le ferraillage ne le peuvent pas. De plus, la faiblesse des prix qui leur sont imposés les pousse à recourir toujours plus à la préfabrication en usine, ce qui accroît le problème d'occupation du personnel. Davantage confrontées à la variabilité que les entreprises générales, les entreprises sous-traitantes recourent logiquement davantage à l'intérim. En période de crise, l'intérim est un amortisseur de choix :

«- Il y a eu un premier grand clash en 94-95. Pas de boulot. Pas de boulot, donc les entreprises se cassent la gueule. (...) L'effectif de FF était descendu à 30.

- 30 embauchés ou...?

- En tout. C'est-à-dire qu'on faisait bosser environ 100 mecs : les 70 intérims, dehors, et sur les 40 [embauchés], il y en avait 10 que j’avais placés à droite à gauche dans d'autres entreprises, en prêt de personnel. ».

\section{[M. Renoir]}

En dépit des discours répétés sur la pénurie de main-d'œuvre, les entreprises de ferraillage, comme les entreprises générales, n'embauchent qu'au compte-gouttes. Elles ne recrutent que ceux qu'elles envisagent de promouvoir à des postes d'encadrement. Voici ce qu'en dit le patron de FE :

«- Je préfère embaucher des gens que je perçois qu'ils vont évoluer au fil des années dans l'entreprise. Bon, tout le monde peut pas devenir chef de chantier, par contre tu sens qu'un mec va devenir ouvrier qualifié, après ouvrier hautement qualifié, après petit chef d'équipe, ça tu le sens. Tu le perçois quand même assez vite. L'idéal, c'est d'embaucher ce type de main-d'œuvre là. Pour moi, parce que comme ça, le conflit social, il existe pas. Dans la mesure où quelqu'un augmente en compétence, moi mon travail c'est de valider les compétences de chacun. Et de valider, au bout d'un moment, par une augmentation de salaire. ».

A ceux qui sont promis à une promotion, le statut d'embauchés; aux «statiques », comme M. Dauby les appelle, le statut éclaté, individualisé et précaire, d'intérimaire. Les premiers sont disciplinés par l'espoir d'occuper des positions supérieures ; les seconds par la menace et l'atomisation. 
3) Car au-delà de la variabilité, il y a la subordination particulière que produit l'intérim, liée à la menace quotidienne d'être renvoyé. Dans la mesure où les conditions de travail sont plus pénibles, car plus dominées, dans le ferraillage, il faut que la subordination soit accentuée, permanente. Selon Daniel Vany, chef de chantier de FF :

«- Un exemple, demain il va m'énerver, je vais le virer. Parce qu'il est intérimaire. J'ai ce pouvoir. C'est un pouvoir, d'ailleurs, qu'on devrait pas avoir. Parce qu'il a faim.

- Mais t'en uses quand même?

- Tout le monde en use.

- Je dis pas le contraire.

- Tout le monde en use, de ce pouvoir. (...) La terreur. Et tu peux pas... C'est rare d'avoir des ouvriers qui n'ont pas peur du chef parce qu'ils connaissent le travail. C'est rare. (...) Et normalement, dans la loi, les intérimaires travaillent par contrat. Qui est-ce qui fait signer le contrat?

- Il n'y en a pas.

- Voilà. Donc on ne suit pas le droit. Qui contrôle ? (...) Le contrôle [de police] qui s'est passé à Roissy, c'est parce qu'un bâtiment s'est cassé la tronche. ».

Les quelques témoignages d'anciens ferrailleurs s'accordent sur le fait que les salaires étaient bien plus proches de ceux des coffreurs à la fin des années 1980. Il était possible de gagner 3000 francs (450€) par semaine en intérim, parfois plus, alors qu'aujourd'hui les mêmes ferrailleurs dépassent péniblement les 300 euros. Daniel Vany porte ce jugement sur le type de travailleurs prêts à accepter les conditions du ferraillage :

«- Et puis c'est un travail sale, c'est salissant, t’as remarqué. T’as vu ? Qui veut le faire maintenant? Qui veut le faire? A part nous, les clochards. On est des clochards. Oh, faut regarder. Va demander à un jeune, maintenant, de faire du ferraillage : "Non". Donc qui est-ce qui fait la ferraille ? (...) C'est tous ceux qui viennent de l'extérieur, tous les émigrés. Plus ou moins clandestins. Qui n'ont aucune notion du travail, en tant que ferrailleurs. Donc on apprend à ces gens-là, dès qu'ils savent travailler, ils vont où ? Ailleurs. Dès qu'ils ont leurs papiers en règle, certains, je parle pas de tous, il s'en vont ailleurs. Si tu regardes bien, il y a beaucoup de clandestins qui travaillent dans la ferraille. Ou d'anciens clandestins. On commence dans la ferraille, on a les papiers, on va faire autre chose. Ça, je peux te le dire. ».

Il n'est pas le seul à le dire; les premiers concernés vont dans le même sens, par exemple dans cette note de terrain : 
Sur un chantier, Houari, un jeune ferrailleur intérimaire vient me conseiller :

«- La ferraille, c'est le pire des métiers, tu devrais faire autre chose. Ça fait cinq ans, je n'ai jamais vu un Français dans la ferraille. Quand tu n'es pas français, d'accord tu es obligé, mais quand tu es français tu peux faire autre chose ». «Ça fait cinq ans que je fais de la ferraille. C'est parce que je suis sans papiers. Là, je viens de me marier, je viens de recevoir le récépissé, j'ai bientôt les papiers... Plus que quelques jours, et je ne reviens plus jamais dans la ferraille. ». Il a bac+3 en Algérie. Ce qu'il va chercher, c'est un emploi en usine (il parle de Citroën à Aulnay) : « Même si c'est payé 1000 ou 2000 francs de moins par mois, je préfère, tu es tranquille. Tu n'es pas cassé à 40 ans. ».

Dans le ferraillage, ce sont les agences d'intérim qui endossent ce rôle de fourniture de sans-papiers ${ }^{1}$. Par conséquent, et dans l'état actuel des contrôles sur le terrain, les utilisateurs n'ont pas à se soucier de qui ils utilisent. Et même si elles voulaient veiller à ne pas utiliser de sans-papiers sur leurs chantiers, les entreprises utilisatrices ne le pourraient peut-être pas - en tout cas, pas dans le cadre décentralisé de gestion des intérimaires qu'elles ont institué. Ce sont en effet les chefs de chantier qui s'occupent de commander et de renvoyer les intérimaires, au jour le jour pour une part d'entre eux. L'intérim n'est rentable qu'à ce prix, celui d'une gestion au plus juste et au plus court que seul un cadre présent sur le chantier peut assurer. Or ces chefs accepteraient difficilement qu'on exige d'eux de contrôler les papiers de leurs ouvriers : parce qu'ils considèrent que ce n'est pas leur métier, que cela fait perdre du temps, et que cela ruinerait entièrement la confiance et la solidarité qu'ils ont pu nouer avec leurs ouvriers ou du moins certains d'entre eux car traquer les sans-papiers risquerait de décimer jusqu'aux noyaux des effectifs. Ainsi parle Armando, chef de chantier de FE:

«- Nous on s'en fout. On demande des gars à l'intérim, ils nous en envoient. Ils envoient les photocopies des papiers des intérimaires à Fanny [la secrétaire de l'entreprise], mais après on s'en fout s'ils ne sont pas bons. Normalement nous aussi on doit prendre les photocopies des papiers d'identité.

- [autre chef de chantier présent pendant une partie de l'entretien] Tu fais ça, toi?

1. Ce point ne sera pas développé. Précisons tout de même que, souvent, les intérimaires sans papiers présentent des faux papiers (que eux comme les commerciaux d'intérim disent grossiers) qui permettent à l'agence de donner le change en cas de contrôle (un ferrailleur : "Ils font des photocopies des papiers qu'on leur donne, et c'est tout. Si jamais il y a un problème, ils montrent la photocopie, ils disent: "Moi je ne savais pas, on m'a donné ça..." "). 
- Oui, je les ai là.

- Moi je ne le fais pas. De Souza [le conducteur de travaux de l'entreprise] m'a dit une fois de vérifier si les papiers des gars sont bons. Je lui ai dit d'aller se faire foutre, si j'avais voulu être flic je serais dans la police. (...)

- Si les papiers sont faux, c'est pas notre problème. Au chantier de la N. [prestigieux magasin parisien], le gardien du chantier les a passés à l'ordinateur : il y avait trente faux papiers sur le chantier, mais qu'est-ce qu'ils allaient faire ? Arrêter le chantier? Ils ont continué le chantier. C'est à l'intérim de vérifier. ».

Pour les jeunes immigrés maghrébins du ferraillage, généralement présents en France depuis moins de dix ans, ce métier constitue un sas qui peut devenir un piège. Ils n'endurent le travail que parce qu'ils comptent le quitter, ou, plus rarement, y gagner des galons. Mais la précarité de l'emploi et la dureté du travail exposent à des périodes d'arrêt de travail qui font reculer d'autant la réalisation des projets et l'obtention de papiers. Et, une fois les papiers obtenus, il n'est pas toujours facile de quitter effectivement le ferraillage, comme on en avait le projet, faute de pouvoir se reconvertir ailleurs. La carte d'un an n'est pas si solide (Thierry, 2001), la précarité du séjour est loin d'être levée. Aussi la frontière entre étrangers réguliers et irréguliers ne sépare pas deux types d'étrangers, deux logiques de séjour foncièrement distinctes; elle est plutôt un rappel à l'ordre de la précarité, pour les irréguliers bien sûr, mais aussi pour les réguliers. Dans le bâtiment, cette proximité des situations se traduit par le fait que des étrangers aux titres de séjour temporaires et des sans-papiers occupent des emplois et des qualifications similaires. Même si l'emploi d'étrangers sans titre de séjour ou sans autorisation de travail nécessiterait une analyse à part, il faut se souvenir que dans la pratique des chantiers, les employeurs ne voient pas là une question fondamentale : il suffit qu'ils sachent globalement leur main-d'œuvre précaire.

\section{Mais pas n'importe lesquels: des assignations ethniques}

On vient de voir que les ferrailleurs, hors chefs, sont très majoritairement des immigrés maghrébins ${ }^{1}$. Voyant cela à mes débuts sur les chantiers, je faisais l'hypothèse que la concentration ethnique était le résultat d'une organisation structurée. Je l'imaginais comme le produit : 1) soit d'une politique d'entreprise, donc d'une organisation centralisée et structurée selon une logique d'apartheid professionnel (selon le modèle Citroën tel que décrit

1. Exception notable d'un chantier où j'ai travaillé durant six semaines, et où le ferraillage n'était pas externalisé, mais pris en charge directement par l'entreprise générale (avec un recours à l'intérim représentant environ un tiers de l'effectif) : le profil des ferrailleurs était plus diversifié qu'ailleurs, au niveau de l'âge comme de l'origine. 
par Robert Linhart [1978], qui construit délibérément des ségrégations ethnico-professionnelles au sein de la grande entreprise) ; 2) soit d'une niche ou d'une entreprise ethnique, que j'identifiais à une structure (de parenté, d'amitié, d'obligations et de protections interpersonnelles) préexistant à l'entrée dans le métier, ou entretenue en dehors du travail. Bref, l'idée était que cette concentration résultait d'une structuration sociale minimale, soit dans un cadre organisé et stable comme une grande entreprise, soit parce que la structure de relations n'était pas liée qu'au travail et renvoyait à des relations hors travail. Or il est apparu que le processus de recrutement et de sélection des ferrailleurs est décentralisé et démultiplié. Entre l'entreprise de ferraillage, le chef de chantier et l'agence d'intérim, trois échelons au moins sont susceptibles d'intervenir dans ce processus. De plus, la mise au travail se fait sous un contrat précaire, selon des mécanismes qui atomisent les individus et instituent entre eux une concurrence universelle. Cette idée de concurrence universelle est contradictoire avec le résultat de la surreprésentation d'une origine ethnique parmi les ferrailleurs. Dans d'autres métiers, ce sont d'autres origines qui sont surreprésentées : Portugais dans le coffrage, Ouest-Africains (Maliens notamment) chez les manœuvres.

Mais en réalité, il n'y a ni politique centralisée et ségrégative, ni structuration préalable. C'est une conjonction d'éléments qui explique la concentration ethnique.

\section{Des différences dans les conditions légales de séjour}

Ferrailleur est l'un des métiers les plus précaires, pénibles et mal rémunérés du bâtiment. Tandis que les noyaux stables et très qualifiés des collectifs de travail sont appelés à avoir une existence sociale qui concorde avec ce profil, les entreprises recherchent en revanche pour les «volants» (on dit aussi les "périphéries ») des personnes légalement et socialement vulnérables. Ce seront des étrangers précaires ou sans papiers. Or les immigrés d'Europe communautaire, et donc notamment les Portugais, ont le droit de circuler et séjourner librement en France - si bien qu'il devient même difficile de parler d'immigrés. Ce statut privilégié, qui date de 1991, prolonge une préférence plus ancienne et moins formalisée de l'Etat français envers la migration portugaise, attestée dès la fin des années 1960 (Henneresse, 1979 ; Viet, 1998), alors que pour les autres origines la politique migratoire se durcit. Cette préférence est liée à des préoccupations spécifiques de l'Etat français (volonté de ne retenir que les migrants « assimilables », européens), mais elle a des conséquences indirectes sur les chantiers : voyant leur position légalement consolidée, les Portugais ont davantage vocation à occuper les postes stables, tandis que les Maghrébins et Africains sont relégués dans les relais de l'externalisation. 


\section{Une gestion raciste de la main-d'œuvre}

Parler de " gestion raciste » des travailleurs est peu courant, ne serait-ce que parce que les catégories de gestion ordinaires en entreprise sont strictement techniques (qualification, ancienneté...) ou bien renvoient à des comportements individuels (compétence, loyauté...), bref disposent d'une apparence universaliste. Une éventuelle " gestion raciste » des travailleurs serait donc souterraine et informelle (Tripier, 1990: 140 et 182). Dans le BTP, elle n'est cependant pas toujours si secrète ${ }^{1}$. Un directeur du recrutement, travaillant au siège d'une des trois majors, expliquait ainsi la discrimination constatée dans son groupe :

«- Je pense que les gens du Maghreb ne raisonnent pas de la même manière que, par exemple, des Portugais ou des gens de l'Est. Ils ont un raisonnement qui n'est pas le même. Les gens du Sud, c'est vrai, du Maghreb surtout, ont une façon de travailler... Les gens des îles, tout ça, c'est des gens qui ont de la difficulté à s'adapter aux rythmes de vie qu'on peut avoir en France aujourd'hui, notamment sur nos chantiers, où il y a une pression de mise assez importante. On a réduit les temps de travail, mais il faut toujours produire autant, il faut... Il y a une qualité du travail à rendre dans un minimum de temps, donc il faut être vif. Il faut être vif, il faut, je dirais, un peu aimer ça, il faut être un peu comme ça. Alors c'est vrai qu'un Portugais est très réactif, très très vif, quitte à faire des bêtises, mais il réagit très très vite. Il fonce. Sans trop réfléchir. On pourrait dire que le Maghrébin c'est l'inverse, il réfléchit peut-être de trop avant de faire les choses, donc voilà. ».

Bien que ce genre de déclarations soit instructif, une difficulté demeure pour qui veut identifier les discriminations directes commises dans le bâtiment. Elle tient moins au caractère souterrain d'une éventuelle gestion raciste mise en place par les entreprises, qu'à une délégation de cette gestion (et même du choix de pratiquer une telle gestion) aux échelons de commandement les moins formels et les moins contraints bureaucratiquement : chef

1. Dans l'une de ses seules enquêtes consacrées aux salariés de la construction, et plus précisément aux salariés immigrés, le journal de référence de la profession, Le Moniteur $\left(n^{\circ} 4511\right.$ 11/05/1990), annonçait dès l'introduction: « [Les dirigeants d'entreprise] ne mettent pas en avant une égalité entre salariés, quelle que soit leur nationalité ou la couleur de leur peau, mais parlent de différences de compétences et d'ambitions qui font bon ménage entre elles ». Tout l'article, basé sur des témoignages de dirigeants, soutient cette vision d'un modèle " ensemble et inégaux ", inversion du célèbre " séparés mais égaux " de la ségrégation américaine, qui se prétend cependant tout aussi harmonieux: "Les dirigeants d'entreprise que nous avons interrogés affichent pas mal de sérénité et évoquent une "cohabitation convenable" ". L'un d'entre eux " décrit "une bonne ambiance, un bon esprit de chantier, sans jamais un seul licenciement pour rixe". II ajoute: "Les Européens profitent de leur situation hiérarchique, par rapport aux Maghrébins, mais ceux-ci sont d'accord, comme s'il s'agissait, pour les uns et pour les autres, d'un ordre normal des choses!" ". 
de chantier, entreprise sous-traitante, agence d'intérim. Prenons l'exemple des agences d'intérim. La directrice d'une d'entre elles (25 ans d'expérience) décrit ainsi ses rationalisations gestionnaires :

«- [Les Maliens] sont des gens passifs, vraiment. Je peux pas parler d'eux parce qu'ils sont franchement, vraiment très gentils, je dirais qu'il y a pas de problème. Pas de problème avec ces gens-là. (...)

- Des Maghrébins, en manœuvres, il y en a ?

- Quelques-uns. Ouais, quelques-uns. J'en prends pas, moi. Très peu. Eux, oui, si j'en prenais, il y aurait des histoires, les gens qui les prennent ont beaucoup d'histoires avec eux. Ce sont des gens qui veulent pas, qui veulent pratiquement pas faire grand chose et qui veulent gagner beaucoup d'argent, donc c'est pas possible dans ce métier. C'est pas possible. N'étant pas raciste, hein, mais il y a des races que je ne prends pas. Les Turcs par exemple, je n'en prends pas. C'est pas une question de racisme, mais je les connais trop bien, quoi. (...) Mais par contre, là on a en ce moment un gars, un plombier, un Péruvien, voyez, j'en avais jamais pris, ou très peu, c'est pas des qualifications, c'est pas des gens qu'on voit beaucoup dans l'intérim. (...) Et bien c'est des gens très gentils, vraiment très respectueux, très gentils, vous voyez, ça c'est une race que je connaissais pas et qui est vraiment parfaite. ».

Le schéma est amené à être explicité quand un candidat n'y correspond pas :

«- Ainsi, alors que je recherche un poste de manœuvre, une autre commerciale me dit : "J'ai quelque chose en démolition, ça serait dès demain. Mais... non, la démolition ce n'est pas pour vous. C'est du marteau piqueur toute la journée. - J'ai déjà fait. - Je ne dis pas que vous ne savez pas faire, mais il y a des trucs plus intelligents. Et c'est vraiment fatigant. Il n'y a que les Maliens qui arrivent à faire ça. ».

Les Portugais «nés dans la maçonnerie », ouvriers exemplaires ; les Africains ou Maliens (les deux termes sont souvent utilisés de manière équivalente), manœuvres dociles ; les Algériens, ferrailleurs, colériques et revendicatifs ; les Français, ouvriers qualifiés « qui se prennent pour des cadors", "picolos », feignants, hargneux : ce sont quelques-unes des images les plus aisément repérables qui circulent dans l'intérim du bâtiment ${ }^{1}$. Derrière un

1. Du moins dans celui du boulevard Magenta à Paris, qui concentre les agences d'intérim du BTP de la région ; car dès lors qu'on réalise le même type d'entretiens dans des agences d'intérim de la rue de la République à Marseille, qui y joue le même rôle, les appréciations racistes demeurent, mais leur contenu se modifie. A Marseille, les Africains d'Afrique subsaharienne sont inexistants, ou du moins, invisibles. Les emplois les moins qualifiés sont occupés presque exclusivement par des immigrés maghrébins. Et du même coup, l'habit du travailleur " serviable ", " gentil ", " bosseur ", " docile " est investi par ces derniers, dont on se plaît à dire à Paris qu'ils sont peu recommandables. 
masque d'assertion définitive, ces stéréotypes doivent une part de leur raison d'être à la compréhension immédiate, circonstancielle, qu'ils cherchent à assurer, et qui n'a pas besoin de dépasser le monde du boulevard Magenta ou de la rue de la République. Cette compréhension figée dans une répartition raciste des tâches déforme et réifie les trajectoires sociales des intérimaires, mais donne un semblant de stabilité informationnelle à l'activité aléatoire que constitue la fourniture de main-d'œuvre. Ce n'est pas un racisme doctrinaire ou dogmatique. Tant que l'activité marche, tant que la marge commerciale de l'agence d'intérim est assurée, les commerciaux d'intérim n'ont aucune raison de changer leurs représentations. Il y a un sens commun partagé entre les agences d'intérim et leurs clients (les entreprises). Aller à l'encontre de ce sens commun, c'est prendre un risque commercial; il vaut donc mieux s'y plier, quand bien même on n'adhère pas à ces représentations. Si l'on recherche un grutier et que le seul qu'on trouve est malien, on finira bien par l'envoyer; mais si un Algérien se présente (les Algériens ayant la réputation d'être de bons grutiers), il a toutes les chances d'être envoyé en priorité. Ces représentations communes sont d'autant plus efficaces qu'elles entretiennent une relation de circularité avec la réalité : puisque les Maliens sont principalement manœuvres, c'est qu'ils ne sont bons qu'à ça, finit-on par se dire (de même pour les Maghrébins ferrailleurs ou les Portugais coffreurs). Par exemple, si un Maghrébin sans qualification se présente à une intérim on l'enverra probablement comme manœuvre en ferraillage ; si c'est un Malien, comme manœuvre tout court. L'intérim conclura que les Maghrébins sont «faits pour» le ferraillage, les Maliens sont « faits pour » être manœuvres. Et elle aura raison puisque c'est elle qui les fait ferrailleurs et manœuvres.

\section{Le recrutement par cooptation}

A FF, le premier conducteur de travaux fut M. Renoir : non pas un homme du métier, mais un commercial ignorant des armatures, qui avait pour principale qualité d'être le gendre du patron, M. Harmann. Ce dernier ne voulait pas d'un technicien, mais d'un homme de confiance. Puis M. Harmann embauche, comme cadres de chantiers, deux immigrés portugais, Paulo et Jacky. Jacky a un beau-fils, Ilinho, arrivé à l'âge de 19 ans en France, au début des années 1990. Il le fait entrer dans l'entreprise. Ilinho croit que c'est juste le temps de gagner un peu d'argent ; en réalité, il s'éternise, en même temps qu'il est choisi par FF pour occuper des positions de chef d'équipe puis, cinq ans seulement après son arrivée dans l'entreprise, de chef de chantier. Son frère suit le même parcours au sein de FF.

A FE, lors du départ en retraite de son associé, M. Dauby choisit l'un de ses chefs de chantier, M. de Souza, pour en faire le conducteur de travaux de l'entreprise. Plusieurs membres de la famille de M. de Souza sont embauchés 
dans l'entreprise, à des postes de cadre : son frère, son neveu Ernesto, puis un cousin et le beau-frère d'Ernesto. En tout, cinq personnes d'une même famille sur un effectif embauché de 24. Etre introduit par un membre de sa famille n'est pas en soi une garantie de réussite (ainsi Ernesto peste-t-il souvent contre les « arnaques » de son oncle), mais elle assure une certaine bienveillance de principe (" mais c'est lui qui m'a donné ma première chance ", précise Ernesto). Sur un chantier de FE, Abdelkader, ferrailleur intérimaire récemment intégré au noyau d'Ernesto, a été introduit par son père (chef de chantier ou chef d'équipe intérimaire selon les périodes, et qui travaille souvent avec FE).

«- Au début je ne savais même pas faire une attache, elles ne tenaient pas. J'avais du mal, vraiment. Au bout de trois semaines, Ernesto m'a arrêté. Mon père est allé le voir, il lui a demandé : "Pourquoi tu l'as arrêté, c'est mon fils, etc." Ernesto a dit: "Je n'ai pas trop de boulot en ce moment, et comme il ne connait pas trop..." Après mon père est venu me voir, il m'a dit: "Attends, une semaine, dix jours, et ils vont te reprendre". Après une semaine, j’ai recommencé, et là ça a été. J'ai commencé à apprendre, à progresser. ».

Un chef d'équipe intérimaire, Mohammed, ferrailleur depuis cinq ans, toujours avec FE, avait de même introduit (mais uniquement sous le statut d'intérimaire) quatre membres de sa famille parmi les ferrailleurs de FE. Leur ancienneté dans le ferraillage et «dans » FE allait de quelques mois à deux ans. M. Dauby, le patron de FE, explique que ce type de regroupement familial est loin d'être désavantageux pour l'entreprise :

«- Il a démarré comme stagiaire chez nous. Après il a franchi toute la pyramide de la hiérarchie. (...) La famille, ils ont faim, ils veulent bosser, il veut en mettre un, deux, trois. Nous on s'en fout, je veux dire, ils travaillent. (...)

- A la limite, ils sont peut-être plus fiables que des gens...?

- Disons que s'ils viennent, quelque part, ils sont tout de même, entre guillemets, un petit peu sous son parrainage, oui. (...) Donc quelque part, s'ils viennent travailler là, ils sont un petit peu sous sa responsabilité, et comme ce sont quand même des gens qui ont un peu le sens de la famille, ils vont pas lui faire de coup vache.».

Sur le chantier où l'équipe de FF était désorganisée par le contrôle de police, je n’ai pas observé de tels liens familiaux parmi les ferrailleurs intérimaires. Toutefois, plusieurs avaient des amis qu'ils appelaient pour les faire venir sur le chantier, à la demande du chef de chantier. Car ce sont les chefs de chantier, plus que l'entreprise elle-même, qui sont les pivots du recrutement et (opération cruciale dans un contexte de précarité et d'incertitude sur les 
savoir-faire) de la fidélisation des ouvriers, ou du moins de certains d'entre eux. Les chefs de chantier se reposent en partie sur l'intérim, mais de préférence, lorsque c'est possible, sur les ouvriers dont ils disposent déjà.

Le recrutement par cooptation forme une discrimination indirecte : les groupes déjà implantés font entrer des proches (famille, amis, recommandations personnelles...), qui sont généralement de la même origine. Inversement, les groupes qui n'ont pas de pionniers installés dans des positions relativement valorisées continuent d'être pénalisés et relégués. Dans le ferraillage, puisque les connaissances personnelles des ouvriers maghrébins sont souvent également des immigrés maghrébins, le résultat est une concentration ethnique. Au niveau des chefs, le recrutement par cooptation continue de favoriser des immigrés portugais ou (plus rarement) leurs descendants et de freiner l'ascension de ferrailleurs maghrébins - quand il n'existe pas une discrimination raciste de principe.

Le recrutement par cooptation n'est une stratégie courante de placement de leurs proches par des salariés que parce qu'il est, d'abord, une stratégie de recrutement privilégiée par les entreprises. Ces dernières en attendent une économie sur la recherche de personnel et une plus grande fiabilité des recrutés. Tous les employeurs, depuis les entreprises générales jusqu'aux agences d'intérim, mettent en place ce mode d'incorporation de main-d'œuvre. Pour certains, la cooptation représente la majorité des recrutements mais, même quand ce n'est pas le cas, comme le dit un DRH, « c'est surtout très important en qualité, c'est-à-dire qu'il y a peu d'échecs ». Les " échecs » redoutés ne sont pas tant un défaut d'aptitudes professionnelles qu'un manque de certaines qualités sociales, notamment la disponibilité, l'obéissance, la loyauté.

\section{Réinjecter des loyautés dans l'ordre marchand}

En instituant la précarité, les employeurs du bâtiment cherchent à discipliner leur main-d'œuvre. Mais au bout d'un moment, cela risque de générer des effets pervers (de leur point de vue) : à l'absence d'engagement de l'employeur (possibilité de renvoi du jour au lendemain par exemple) risque de répondre celle du salarié (absentéisme, démissions impromptues, mauvaise volonté, etc.). Car la production d'énergie humaine n'est pas comparable à celle d'une machine ; elle nécessite un minimum de loyauté, de fiabilité, que les salariés prodiguent d'autant moins qu'on les trompe et qu'on les précarise. La contestation n'est pas visible au premier abord, car elle est éclatée et peu formalisée. Elle prend surtout la forme de la défection. Si l'on peut changer de chantier ou d'entreprise pour quelques euros, comment maintenir une équipe où s'établit un minimum de coopération et de routine? Si 
ceux qui connaissent le métier fuient ses mauvaises conditions d'exercice (dès lors qu'ils ont des papiers par exemple), que reste-t-il de savoir-faire ? Il fuit. A moins de modifier toute l'architecture du système, par exemple remplacer les actuelles PME du ferraillage par des acteurs étrangers par le biais de la prestation transfrontalière de services, il faut des mécanismes de compensation. Il faut pouvoir générer de la loyauté, là où l'ordre marchand tend à la détruire. C'est pourquoi d'autres liens que les liens salariaux sont mobilisés sur les chantiers : allégeances personnelles, parenté, ethnicité.

Ainsi tous les chefs de chantier (il en est de même des commerciaux d'intérim) ont-ils leur noyau, des ferrailleurs avec lesquels ils ont établi des habitudes et une certaine confiance. Formellement, tous les ferrailleurs sont sous le même statut, intérimaires. Dans la pratique, certains sont et se savent plus stables que d'autres. Cela n'anéantit pas la menace du renvoi du jour au lendemain. Au contraire, c'est cette menace qui permet au chef de chantier d'établir un régime de faveur pour quelques-uns : contre la promesse (même implicite) d'être le dernier licencié, ces quelques-uns prodiguent une plus grande loyauté.

Par exemple, quelque temps après avoir travaillé sur un chantier, je m'apprête à y interviewer Armando (le chef de chantier ferraillage) dans son bureau, quand Kaïs, un ferrailleur intérimaire qui travaille avec Armando depuis des années, entre dans la baraque. Armando lui explique des tâches à effectuer, lui montrant des croquis qu'il a dessinés. Puis le prévient :

«- Je vais devoir renvoyer des gars, on commence à être trop. Le bâtiment 3 est presque fini, le bâtiment 2 aussi. Je vais devoir renvoyer trois gars je pense, on va essayer de les mettre sur d'autres chantiers. Abdel, le type qui travaille avec toi et le petit avec Karim [tous arrivés récemment]. C'est bon maintenant, ils se débrouillent? - Oui, c'est bon, ils peuvent se débrouiller, c'est des ferrailleurs. - Bon, alors c'est bon. Donc il y en a trois qui vont partir, on va essayer de les mettre ailleurs, mais tu ne leur dis rien pour l'instant, $\mathrm{OK}$ ? ».

Par son statut d'emploi, Kaïs n'est pas plus que les ferrailleurs qui seront renvoyés. Mais dans la pratique, il est évident pour tout le monde qu'il est autant, voire davantage, un collaborateur du chef qu'un pair des ferrailleurs présents temporairement sur le chantier.

Lorsque je suis arrivé sur le chantier où le ferraillage était dirigé par Ernesto, ce dernier venait de renvoyer près de la moitié de l'effectif et n'avait conservé que quatre ferrailleurs, ceux avec lesquels il travaille de chantier en chantier ; si un creux d'activité avait dû l'amener à réduire encore l'effectif, il aurait essayé de placer l'un d'entre eux sur le chantier d'un collègue plutôt que de le renvoyer. Sur le chantier de Roissy, où l'effectif était désorganisé, le 
chef disait qu'« avant que les flics viennent, on avait une bonne équipe, ça roulait tout seul », ce que confirmait M. Renoir en expliquant que plusieurs des ferrailleurs qui ont alors disparu travaillaient avec FF depuis cinq ou six ans.

Avec la question de la réinjection de loyauté dans les relations de travail, c'est le caractère particulier de l'échange de force de travail qui est soulevé. Même la théorie économique orthodoxe, qui voudrait n'y voir qu'un échange comme un autre, soumis aux mêmes lois du marché, a fini par admettre sa spécificité. Elle s'est avisée que la forme du contrat de travail pouvait créer une défiance entre employeur et salarié, et que cette défiance réciproque pouvait conduire à un équilibre sous-optimal (de mauvaises conditions de travail contre un travail mal fait). La solution a été baptisée « théorie des contrats implicites ». Elle repose sur l'hypothèse d'une régulation qui subvertit partiellement la logique marchande. Elle montre qu'il peut être avantageux pour les deux parties de maintenir de meilleures conditions de travail que celles déterminées par le marché : le patron y gagne la stabilité de son salarié, et le salarié la stabilité de son emploi. Ce qui s'échange, ce n'est plus seulement un salaire contre de la force de travail, mais une loyauté réciproque : "l'“objet" échangé est non pas une marchandise, mais un ensemble d'engagements mutuels » (Garnier, 1986: 313). La solution est astucieuse mais, en cherchant à préserver le cadre théorique d'ensemble (individualisme méthodologique et contractualisme), elle en révèle les contradictions. Avec l'expression de « contrat implicite », on frôle l'oxymore, si l'on considère que le contrat est au contraire le royaume de l'explicite, de l'enregistrement d'engagements en bonne et due forme. Il s'agit moins d'un concept explicatif que d'un aveu d'impuissance théorique, une boite noire qui est une invitation à l'empirie. De retour sur le terrain, la vision contractualiste est battue en brèche par la réalité de rapports de force inégaux, comme on l'a vu ici. Et, autour de la boîte noire les questions affluent : que recouvre l'« implicite »? Qu'est-ce qui donne à croire à des individus, employeurs et salariés, qu'ils s'entendent sur des choses qu'ils n'expriment pas, qu'est-ce qui peut fonder les allégeances tacites?

Il y a d'une part ces noyaux, relations informelles que tissent tous les individus tenant une part de la gestion de la main-d'œuvre sur le terrain (chefs de chantier, commerciaux d'intérim, petits patrons). En échange d'une gratification, d'une faveur (prime, relative sécurité de l'emploi...), le salarié est fidélisé, tenu par une obligation morale qui sera d'autant plus solide qu'elle pourra se prévaloir de référents extérieurs au travail (notamment familiaux).

Il y a d'autre part les discriminations racistes. Le lien entre ces dernières et les « contrats implicites » peut sembler obscur. Mais dès lors qu'elles sont stabilisées et largement connues, elles peuvent influer sur le comportement 
au travail de leurs bénéficiaires, comme de leurs perdants. Les différentes formes de discrimination sont une toile de fond de l'implicite. Un ouvrier portugais (éventuellement introduit par un proche) s'imagine (et il a raison) un avenir plus prometteur qu'un ouvrier africain. Sans doute cette promesse peut être déçue, sans doute bien des vies d'ouvriers portugais sont traversées de trahisons d'employeurs, de déceptions et de précarités. Mais il est question ici de probabilités : il y a moins de chances pour un ouvrier portugais d'affronter de telles déconvenues. Chances que l'on peut reconstituer abstraitement, par exemple par des statistiques ; mais chances qui sont aussi palpables, sensibles dans le quotidien des chantiers, sédimentées et intériorisées dans l'expérience des individus, qui créent des anticipations et des comportements correspondants. C'est ici peut-être que se rejoignent les attentes des employeurs et celles des salariés : les premiers sont prêts à favoriser une fraction des seconds ; les ouvriers privilégiés s'investissent dans leur travail avec une loyauté à la mesure de leurs espoirs ; et c'est ainsi qu'ils méritent la confiance qu'on leur a accordée. Cependant que d'autres partaient perdants (et en termes de probabilités on peut penser que c'est à raison), et ont pu, par leur comportement, confirmer le verdict qui pesait déjà sur eux.

\section{Conclusion}

Ce qu'enseigne l'étude du ferraillage, c'est que là où la précarité est poussée, des mécanismes informels de compensation sont créés : fidélisation sélective d'intérimaires, recouvrement des relations de travail avec des liens personnels et notamment familiaux, mise en avant de certaines origines ethniques et relégation d'autres. Phénomènes qui n'ont rien de systématique, mais dont la fréquence est suffisamment attestée pour qu'on y voie un trait décisif de la gestion et la mobilisation de la main-d'œuvre dans le bâtiment. Qui n'ont rien de systématique, précisément parce que le système demeure celui du marché, et que les loyautés informelles, les protections sélectives, sont des mécanismes de compensation partielle de ce système. La question n'est pas donc de déterminer si le travail salarié dans le bâtiment est tout entier orienté par la précarité, les recrutements impersonnels, la concurrence entre les travailleurs, la mise au travail basée sur la seule relation marchande ; ou si, au contraire, ce sont le paternalisme, les recrutements sélectifs et protégés, les connivences et le favoritisme, les loyautés et dettes personnelles, les agrégations et discriminations ethniques qui dominent. Il s'agit plutôt de comprendre comment la tension pratique et permanente entre ces deux pôles permet d'assurer, bon an mal an, la production de bâtiments. 


\section{Références bibliographiques}

Burawoy Michael (2003), L'étude de cas élargie. Une approche réflexive, historique et comparée de l'enquête de terrain, 1 ère éd. 1998, reproduit in Céfaï, L'enquête de terrain, Paris, La Découverte/M.A.U.S.S., p. 425-464.

Campagnac Elisabeth (dir.) (1992), Les grands groupes de la construction : de nouveaux acteurs urbains, Paris, L'Harmattan.

Campinos-Dubernet Myriam (1984), Emploi et gestion de la main-d'œuvre dans le BTP. Mutations de l'après-guerre à la crise, Paris, La Documentation française, $350 \mathrm{p}$.

Garnier Olivier (1986), « La théorie néo-classique face au contrat de travail : de la " main invisible » à la " poignée de main invisible »", in Salais, Robert et Thévenot, Laurent, Le travail. Marchés, règles, conventions, Paris, Economica, p. 313-331.

Henneresse Marie-Claude (1979), Le patronat et la politique française d'immigration 1945-1975, Paris, IEP Paris, Thèse de doctorat en Etudes politiques, $610 \mathrm{p}$.

Jounin Nicolas (2006), Loyautés incertaines, les travailleurs du bâtiment entre discrimination et précarité, Thèse de doctorat en sociologie, université Paris VII, $619 \mathrm{p}$.

Linhart Robert (1978), L'établi, Paris, Editions de Minuit, 180 p.

Tallard Michèle (1986), Le statut des salariés du BTP : enjeux et stratégies, Paris, PCA, $239 \mathrm{p}$.

Thierry Xavier (2001), « La fréquence de renouvellement des premiers titres de séjour », Population, 56 (3), 2001, p. 451-468.

Tripier Maryse (1990), L'immigration dans la classe ouvrière en France, Paris, CIEMI-L'Harmattan, 1 ère éd. 1987, 332 p.

Viet Vincent (1998), La France immigrée. Construction d'une politique 1914-1997, Paris, Fayard, $550 \mathrm{p}$. 
